\title{
MEASUREMENT OF DISSOLVED CARBON DIOXIDE CONCENTRATION IN A SURF ZONE
}

\author{
Junichi Otsuka ${ }^{1}$, Yasunori Watanabe ${ }^{2}$ and Ayumi Saruwatari ${ }^{3}$
}

\begin{abstract}
In this study, we measured dissolved carbon dioxide $\left(\mathrm{D}-\mathrm{CO}_{2}\right)$ concentration in a surf zone in a laboratory wave flume filled with freshwater and seawater using a glass electrode $\mathrm{CO}_{2}$ meter, and also observed the air-water turbulent flow field using Particle Image Velocimetry (PIV). D- $\mathrm{CO}_{2}$ concentration increased with time and the bore region reached a saturated state earlier than the transition region. The gas transfer velocity in the transition region was much higher than that in the bore region since the numerous entrained bubbles trapped within three-dimensional vortices significantly contribute to the gas dissolution into water in the transition region. The gas transfer velocity in a surf zone in freshwater were found to be higher than those in seawater. We estimated the gas transfer velocity in a surf zone from the turbulent energy in breaking waves and the Schmidt number. It was found that the gas transfer velocity could be roughly estimated from the turbulent energy in breaking waves.
\end{abstract}

Keywords: breaking waves, gas dissolution, gas transfer velocity, entrained bubbles

\section{INTRODUCTION}

The gas transfer velocity across the air-sea interface over the ocean has been conventionally estimated using models parameterized in terms of wind speed (e.g., Liss \& Merlivat, 1986; Wanninkhof, 1992). The breaking wave effects on the gas transfer into water, such as the aeration effect and the turbulent intensity (Melville, 1996), are included in wind speed parameters. Thus, we cannot apply the wind-speed-based model to the estimation of the gas transfer velocity in a near-shore wave breaking field, where waves break while propagating from offshore to near-shore shallow waters.

In a surf zone, plunging jets of breaking waves sequentially splash on a forward water surface to entrain a number of air bubbles, and to produce three-dimensional vortices (Nadaoka et al., 1989), forming three-dimensional air-water turbulent flow. The entrained bubbles increase the total area of airsea interface per unit volume and enhance the gas dissolution into water. Watanabe et al. (2008) measured the dissolved carbon dioxide $\left(\mathrm{D}-\mathrm{CO}_{2}\right)$ in breaking waves using a two-color laser induced fluorescence technique, and presented that the distributions of the entrained bubbles in breaking waves highly correlated with the $\mathrm{D}-\mathrm{CO}_{2}$ concentration, and the strong turbulence generated by the threedimensional vortices diffused the bubbles and $\mathrm{D}-\mathrm{CO}_{2}$ in a deeper water region. Therefore, to develop an appropriate model to estimate the gas transfer velocity in a surf zone, we need to characterize the gas dissolution in a surf zone on the basis of the bubble transport process and the transition of the turbulent intensity in breaking waves.

In this study, we investigated the temporal variations in the cross-shore distribution of D- $\mathrm{CO}_{2}$ concentration in a laboratory wave flume using a glass electrode $\mathrm{CO}_{2}$ meter to estimate the gas transfer velocity in a surf zone. We also observed the bubble-water turbulent flow field in a surf zone using Particle Image Velocimetry (PIV), and estimated the gas transfer velocity from the turbulent energy in breaking waves.

\section{EXPERIMENTAL SET UP AND CONDITIONS FOR D-CO2 MEASUREMENTS}

Experiments were performed in a wave flume of $8.00 \mathrm{~m}$ in length, $0.60 \mathrm{~m}$ in depth and $0.25 \mathrm{~m}$ in width with transparent acrylic side walls, bottom and cover (see Figure 1). Waves were generated by a piston-type wave generator set up at one end of the wave flume. A hinge was set at the bottom under the wave paddle, and the flume was sloped using an oil jack fixed under the bottom at the other end of the flume to create a 1 in 20 and 1 in 15 beach slope. A compressed $\mathrm{CO}_{2}$ cylinder was connected to the top cover of the flume to supply $\mathrm{CO}_{2}$ in the gas phase over the wave interfaces. The D- $\mathrm{CO}_{2}$ concentration was measured from the wave breaking points to the shoreline at $0.30 \mathrm{~m}$ spacing with a glass electrode $\mathrm{CO}_{2}$ meter (TOA-DKK CGP-1). The measurement times were $1,2,5,10$ and 15 minutes after the first-wave generation. The water surface elevation at each measurement point was measured with a capacitance-type wave gauge. The wave data acquisition frequency was $50 \mathrm{~Hz}$.

Experimental conditions are shown in Table 1 , in which $T$ is wave period, $H_{b}$ is the breaking wave height and $h_{b}$ is the breaking water depth. The wave breaking types were spilling breaker (Case 1, Case 2) and plunging breaker (Case 3, Case 4). The water depth in front of the wave generator was 0.25

\footnotetext{
${ }^{1}$ Civil Engineering Research Institute for Cold Region, Hiragishi 1-3-1, Sapporo, 060 8628, Japan

${ }^{2}$ School of Engineering, Hokkaido University, North 13 West 8, Sapporo, 060 8628, Japan

${ }^{3}$ School of Engineering, Hokkaido University, North 13 West 8, Sapporo, 060 8628, Japan
} 
(a) Side view

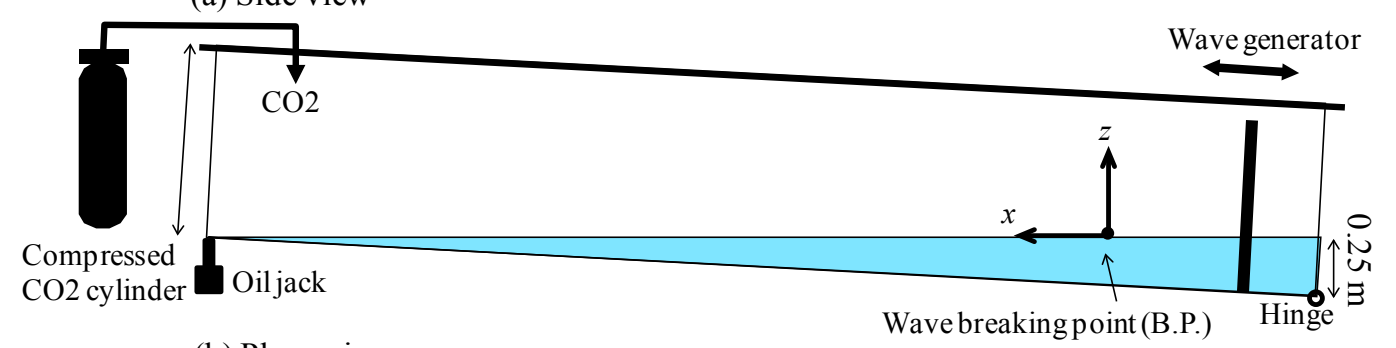

(b) Plane view

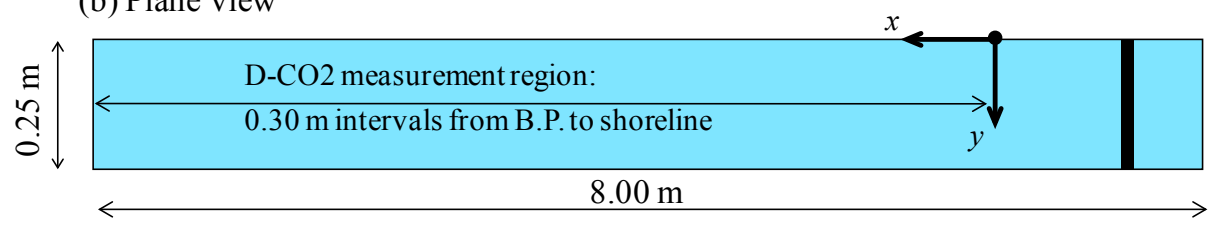

Figure 1. Experimental set up for D-CO2 measurements.

Table 1. Experimental conditions for D-CO2 measurements (T: wave period, $H_{b}$ : breaking wave height, $h_{b}$ : breaking water depth).

\begin{tabular}{|c|c|c|c|c|c|c|}
\hline CASE & $\begin{array}{c}T \\
(\mathrm{~s})\end{array}$ & $\begin{array}{c}H_{b} \\
(\mathrm{~cm})\end{array}$ & $\begin{array}{c}h_{b} \\
(\mathrm{~cm})\end{array}$ & $\begin{array}{l}\text { Bottom } \\
\text { slope }\end{array}$ & $\begin{array}{c}\text { Breaker } \\
\text { type }\end{array}$ & Media \\
\hline CASE 1 & \multirow{2}{*}{1.4} & \multirow{2}{*}{13.6} & \multirow{2}{*}{17.5} & \multirow{2}{*}{$1 / 20$} & \multirow{2}{*}{ Spilling } & Freshwater \\
\hline CASE 2 & & & & & & Seawater \\
\hline $\begin{array}{l}\text { CASE } 3 \\
\text { CASE } 4\end{array}$ & 1.9 & 14.5 & 15.0 & $1 / 15$ & Plunging & $\begin{array}{c}\text { Freshwater } \\
\text { Seawater }\end{array}$ \\
\hline
\end{tabular}

(a) Side view

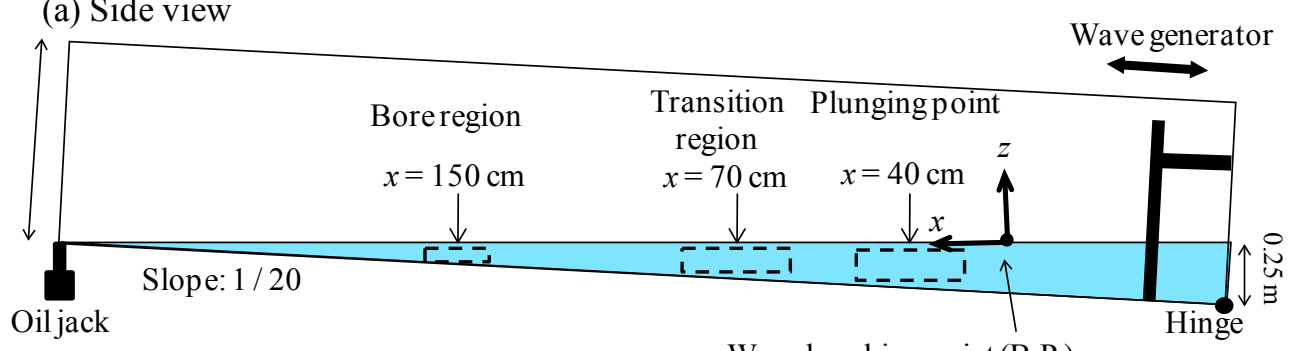

(b) Plane view

Wave breaking point (B.P.)

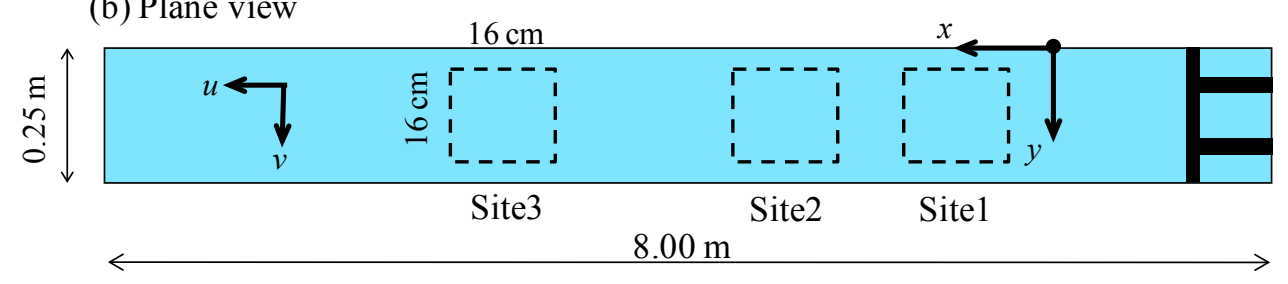

Figure 2. Experimental set up for PIV measurements.

$\mathrm{m}$ in all cases. The flume was filled with freshwater (Case1, Case3) and seawater (Case2, Case4). In all the experiments, the water temperature was observed to vary within a very narrow range $\left(20 \pm 0.5^{\circ} \mathrm{C}\right)$.

\section{EXPERIMENTAL SET UP AND CONDITIONS FOR VELOCITY MEASUREMENTS}

The velocity fields of bubbler-water turbulent flow under breaking waves were measured using Particle Image Velocimetry (PIV) on the horizontal plane and vertical plane crossing the beach in the center of the flume (see Figure 2). The measurements were performed in the same flume used in the D$\mathrm{CO} 2$ measurements, which was filled with freshwater and was sloped 1 in $20 . T, H_{b}, h_{b}$, and the wave 
(a) Plane view

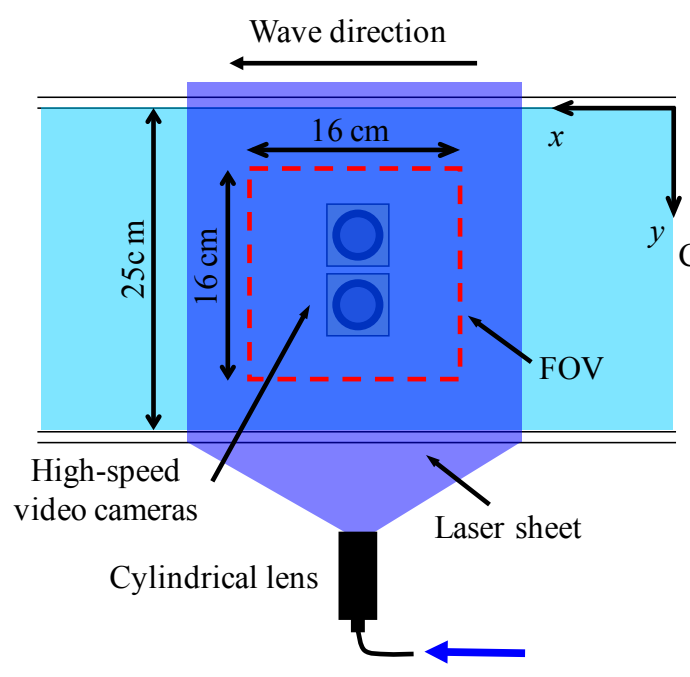

Argon laser

(Wave length: 514.5 \& $488 \mathrm{~nm}$ ) (b) Cross-section view

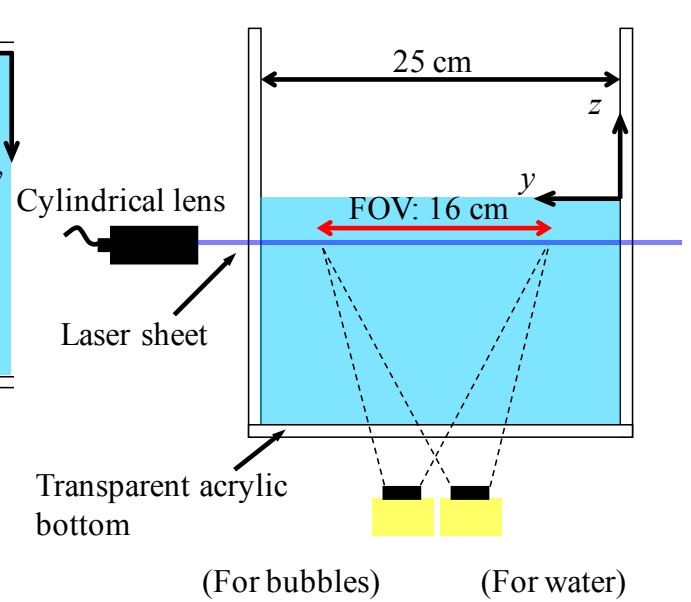

High-speed video cameras ( 8 bit, $250 \mathrm{fps}, 1 \mathrm{~K} \times 1 \mathrm{~K}$ pixels)

Figure 3. Location of high-speed video cameras and laser sheet for PIV measurements on the horizontal plane ((a): plane view, (b): cross-section view).

(a) Side view

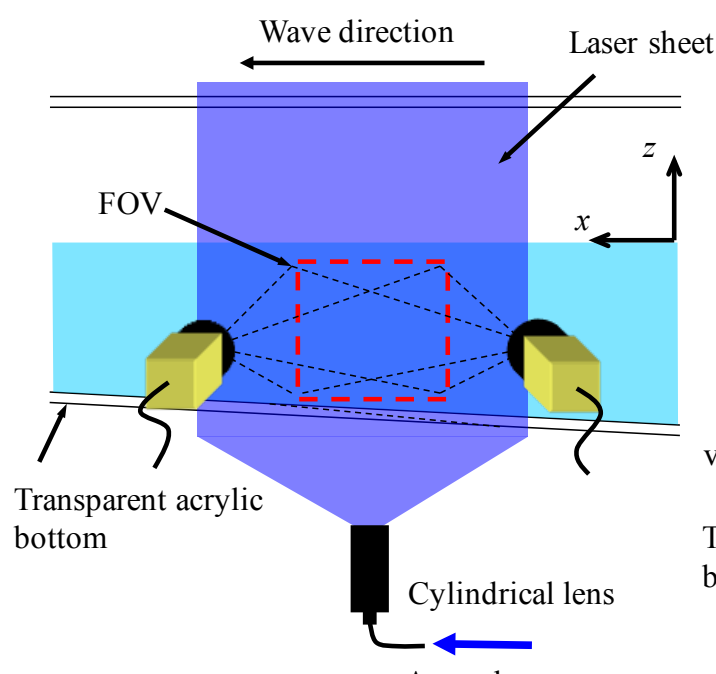

Argon laser

(Wave length: $514.5 \& 488 \mathrm{~nm}$ ) (b) Cross-section view

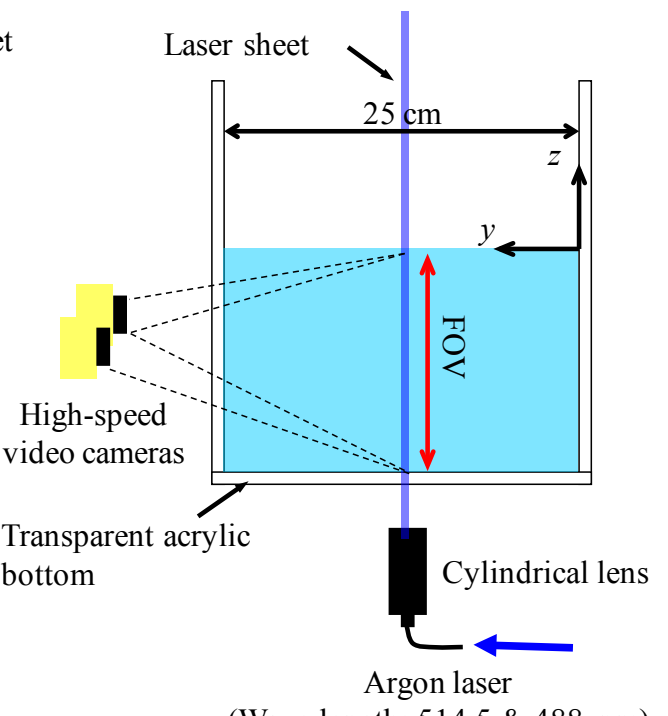

(Wave length: $514.5 \& 488 \mathrm{~nm}$ )

Figure 4. Location of high-speed video cameras and laser sheet for PIV measurements on the vertical plane ((a): side view, (b): cross-section view).

breaking type were $1.4 \mathrm{~s}, 13.6 \mathrm{~cm}, 17.5 \mathrm{~cm}$ and spilling breaker, respectively. In the case of the measurements on the horizontal plane, an argon-laser sheet was horizontally emitted from the side of the flume. The water and bubble flows on the horizontal laser sheet were simultaneously recorded using two 8-bit high-speed video cameras located under the bottom of the flume (see Figure 3). In the case of the measurement on the vertical plane, the laser sheet was vertically emitted from the bottom to the free surface, and the two cameras were located at the side of the flume (see Figure 4). The acquisition frequency was $250 \mathrm{~Hz}$, and the resolution of the camera was $1024 \times 1024$ pixels. The field of view for each camera was $16 \times 16 \mathrm{~cm}^{2}$. The image acquisition system was triggered by a TTL signal 


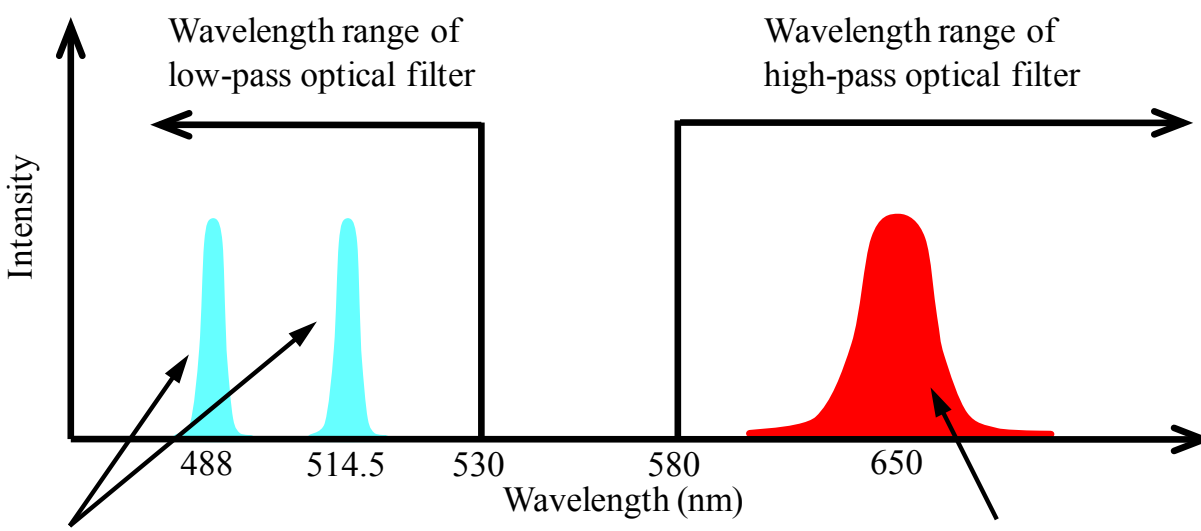

Spectrum of reflection light from bubbles

Spectrum of fluorescent light

Figure 5. Wavelength range of high-pass and low-pass optical filters.

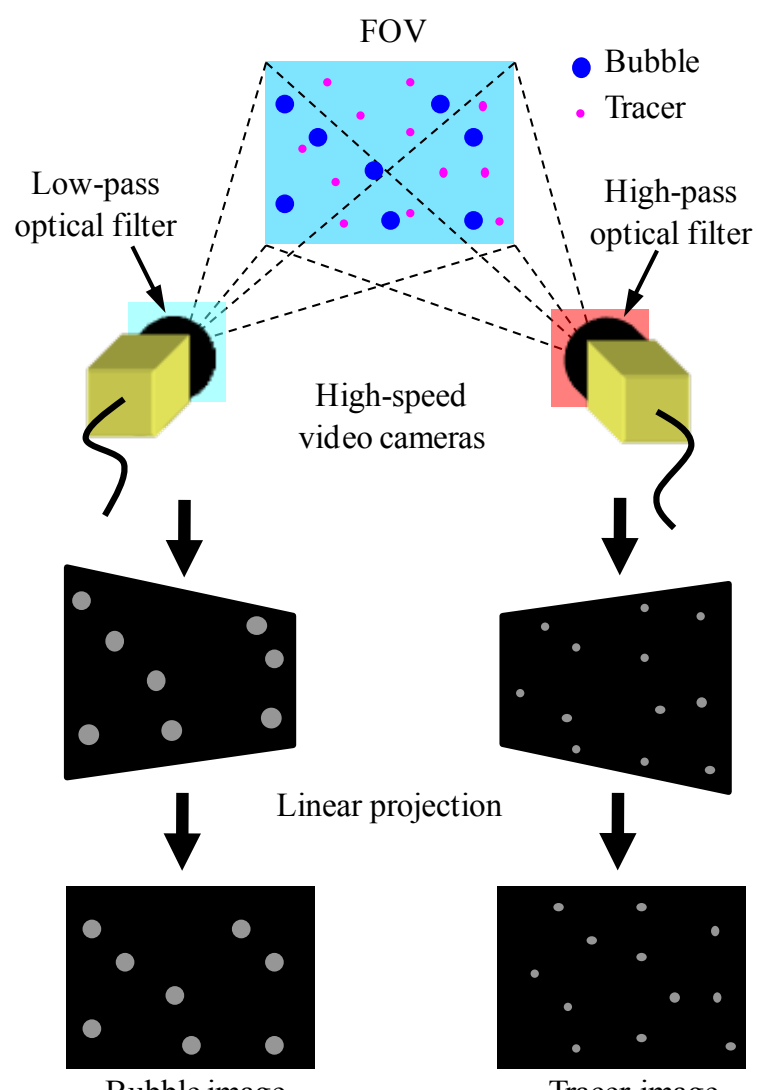

Bubble image

Tracer image

Transformed to real coordinates

Figure 6. Simultaneous image acquisition procedure for water and bubble flows using an optical filtering technique.

generated when a wave gauge placed in front of the wave paddle detected the first wave. Another wave gauge was set at a measurement site to be triggered by the same signal as the image acquisition system. Simultaneous velocities and wave fields were obtained at the same site by the synchronized images and wave records. The wave data acquisition frequency was $50 \mathrm{~Hz}$.

The water was seeded with fluorescent neutral buoyant tracers of $50-110 \mu \mathrm{m}$ in diameter. A highpass optical filter (a wavelength of $580 \mathrm{~nm}$ or shorter being filtered out) was attached on one camera 

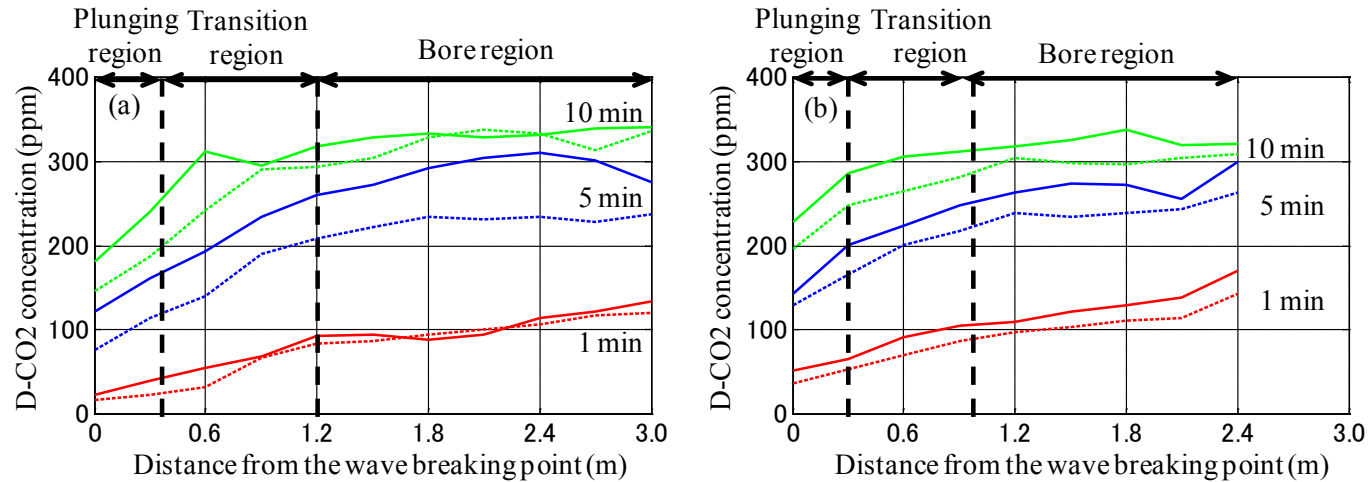

Figure 7. Temporal variations in the cross-shore distribution of D-CO2 concentration ((a): spilling breaker, CASE 1 (solid line, freshwater), CASE 2 (dotted line, seawater), (b): plunging breaker, CASE 3 (solid line, freshwater), CASE 4 (dotted line, seawater), red: $1 \mathrm{~min}$, blue: $5 \mathrm{~min}$, green: $10 \mathrm{~min}$ after the first wave generation).
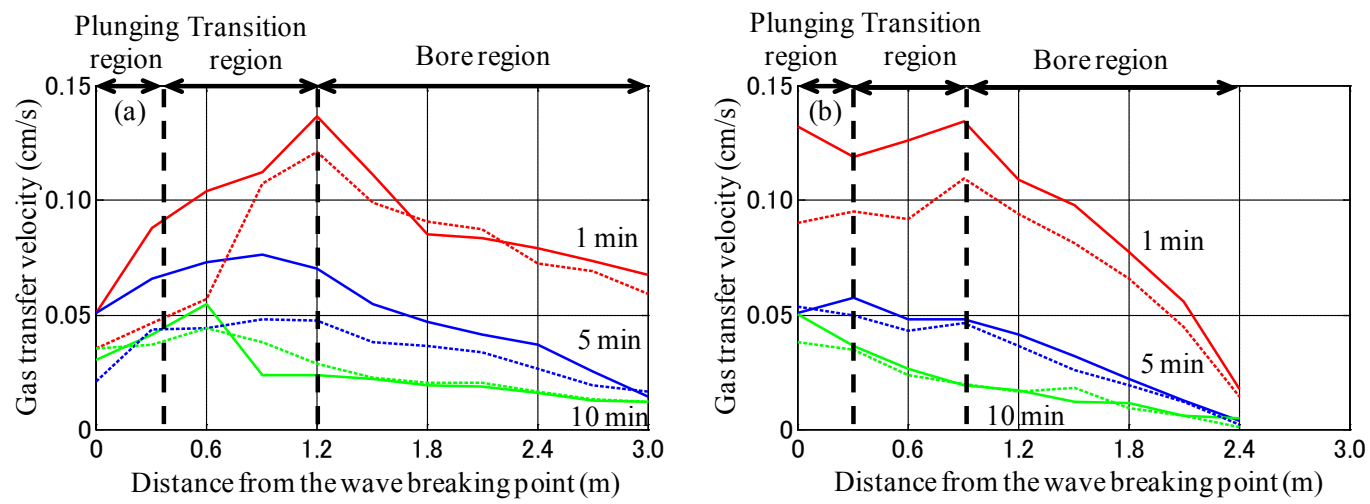

Figure 8. Temporal variations in the cross-shore distribution of gas transfer velocity ((a): spilling breaker, CASE 1 (solid line, freshwater), CASE 2 (dotted line, seawater), (b): plunging breaker, CASE 3 (solid line, freshwater), CASE 4 (dotted line, seawater), red: $1 \mathrm{~min}$, blue: $5 \mathrm{~min}$, green: $10 \mathrm{~min}$ after the first wave generation).

lens to capture only the laser-induced fluorescence lights at a peak wave length of $650 \mathrm{~nm}$ from the tracers, and to filter out reflection lights (a wavelength of $514.5 \mathrm{~nm}$ and $488 \mathrm{~nm}$ ) from entrained bubbles (see Figure 5, Figure 6). A low-pass optical filter (a wavelength of about $530 \mathrm{~nm}$ or longer being filtered out) was also attached on the second camera lens to capture only the reflection lights from the bubbles (a wavelength of $514.5 \mathrm{~nm}$ and $488 \mathrm{~nm}$ ) and to filter out the fluorescent lights (see Figure 5, Figure 6). Image noise was reduced with a Gaussian digital filter. The coordinates of the captured images were transformed to the real coordinates using a linear projection (see Figure 6).

The horizontal $x$ (in the direction of wave propagation), spanwise $y$ and vertical $z$ coordinates with the origin at the breaking point and side wall on a still water level were defined. The measurements were conducted at three sites: site 1 at a plunging point $(x=40 \mathrm{~cm})$, site 2 in a transition region $(x=70$ $\mathrm{cm})$ and site 3 in a bore region $(x=150 \mathrm{~cm})$. The water depths at the three sites were $15.5 \mathrm{~cm}, 14.0 \mathrm{~cm}$ and $10.0 \mathrm{~cm}$, respectively.

Although bubbles can be optically eliminated from images, the measurements could become inaccurate in highly aerated regions due to changes in and obstructions to the optical paths. Therefore, in the PIV experiments, the measurements were limited to non-aerated or diluted bubble flows beneath the wave trough level. A standard type of FFT based PIV technique was used. The window size was $100 \times 100$ pixels $\left(17 \times 17 \mathrm{~mm}^{2}\right)$ and the size of interrogation region was $180 \times 180$ pixels $(31 \times 31$ $\mathrm{mm}^{2}$ ). The window was overlapped at 10 pixels interval.

\section{RESULTS}

Figure 7 shows the temporal variations in the cross-shore distribution of $\mathrm{D}-\mathrm{CO}_{2}$ concentration. D- 


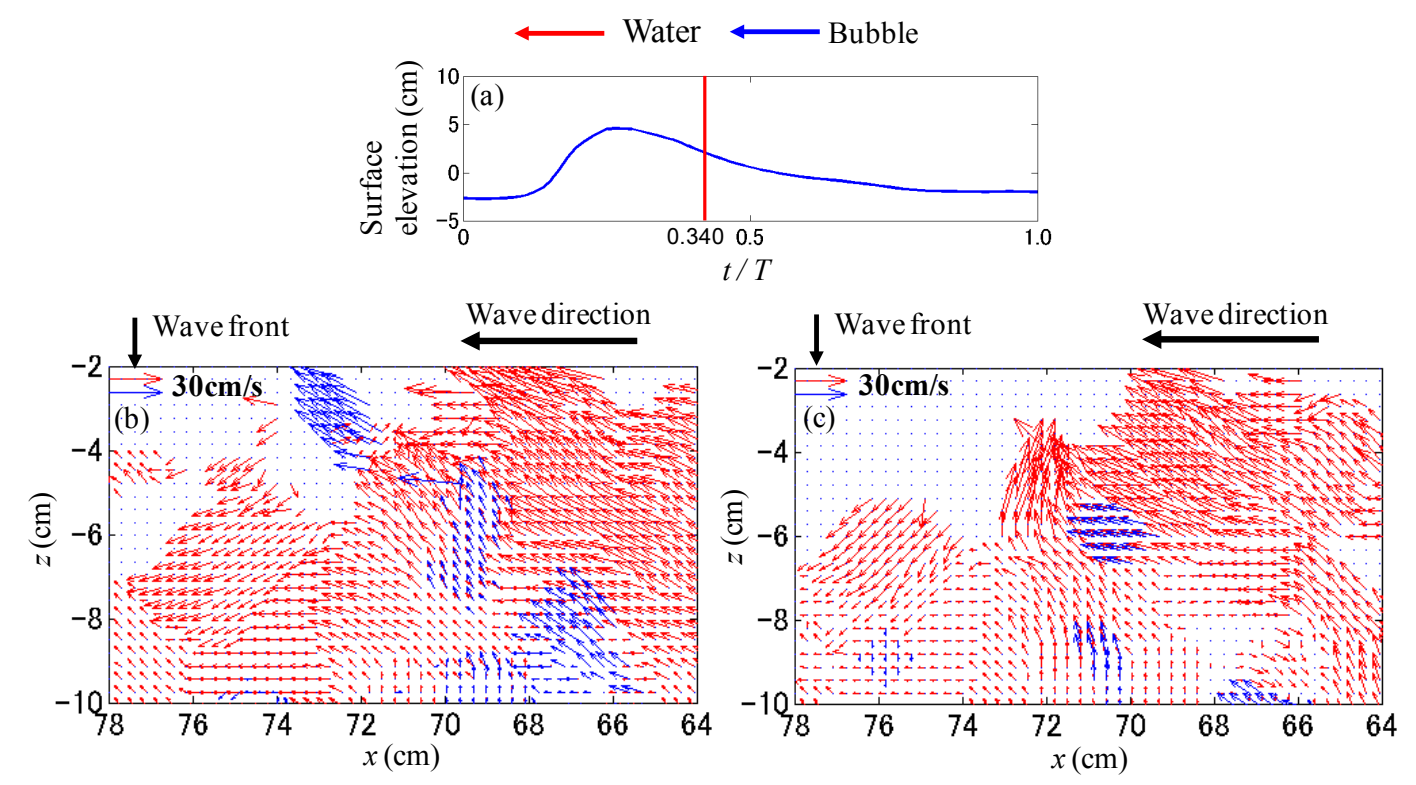

Figure 9. Surface elevation (a) and instantaneous velocities of water and bubble flows on the vertical plane after a breaking wave front has passed over site 2 ((b), (c): successive velocity vector plots from $t / T=$ 0.340 to $t / T=0.343$, red: water velocity, blue: bubble velocity).

$\mathrm{CO}_{2}$ concentration over the whole measurement region increases with time, and the concentration in the bore region becomes almost constant within 10 min after the first wave generation (green lines in Figure 7). Since the water depth in the bore region is shallow, the bore region reaches the saturated state of the gas dissolution earlier than the transition region. In the plunging breaker, a number of entrained bubbles are produced beneath the plunging point of breaking waves to enhance the gas dissolution across the bubble-water interface. Therefore, the $\mathrm{D}-\mathrm{CO}_{2}$ concentration at the plunging region in the plunging breaker is higher than that in the spilling breaker. The concentration in freshwater tends to be higher than that in seawater.

Figure 8 shows the temporal variations in the cross-shore distribution of gas transfer velocity. In this study, the gas transfer velocity at each $\mathrm{D}-\mathrm{CO}_{2}$ measurement point was estimated as shown below. Gas flux $F$ can be expressed as following equation:

$$
F=U \Delta C
$$

where $U$ is the gas transfer velocity $(\mathrm{cm} / \mathrm{s})$ and $\Delta C$ is the difference of gas concentration across the sea surface (ppm). When we assume the region above the wave trough level is saturated, $\Delta C$ is given by:

$$
\Delta C=C_{s}-C_{f}
$$

where $C_{s}$ is the saturated concentration (ppm), $C_{f}$ is the concentration under the wave trough level (ppm). Gas flux also can be given by:

$$
F=\frac{d C}{d t} \Delta z
$$

where $\Delta z$ is the turbulent mixing depth in breaking waves $(\mathrm{cm})$. In this study, we assumed $\Delta z$ is approximately equal to the wave height $H(\mathrm{~cm})$ at each $\mathrm{D}-\mathrm{CO}_{2}$ measurement point. From Eq. 1 to Eq. 3, the gas transfer velocity $U$ is obtained with the following equation:

$$
U=\frac{d C}{d t} \frac{H}{C_{s}-C_{f}}
$$

The gas transfer velocity indicates a high value within $1 \mathrm{~min}$ after the first wave generation (red lines in Figure 8). Since the D- $\mathrm{CO}_{2}$ concentration has not been saturated in short duration after the first 

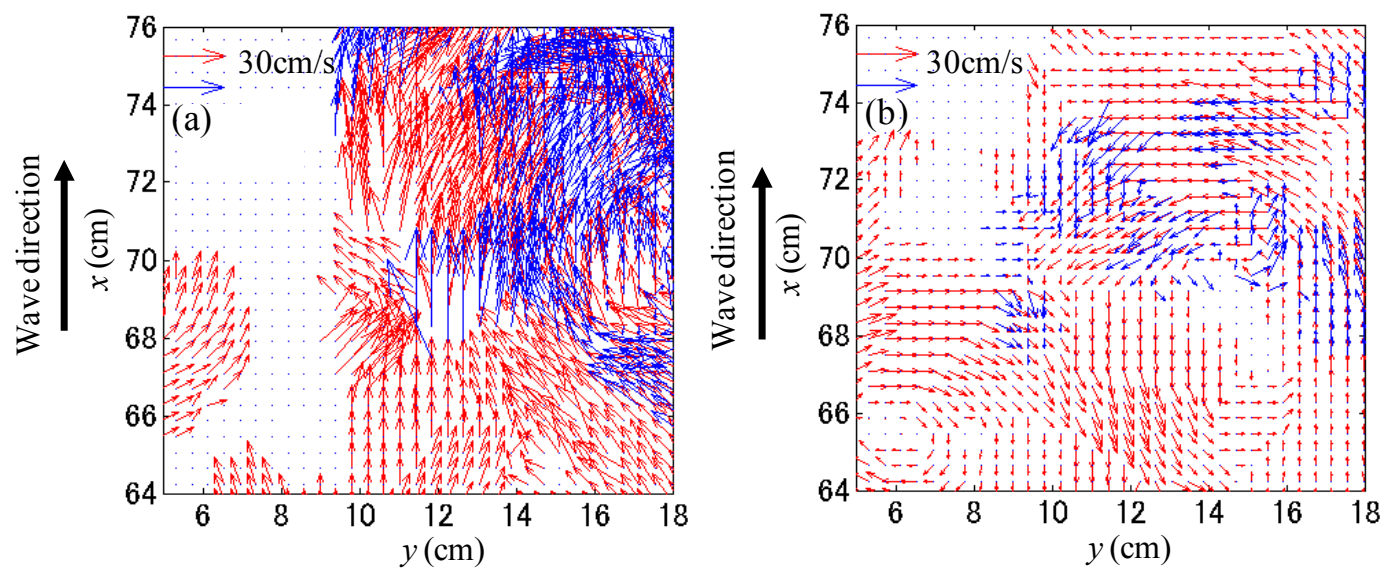

Figure 10. Instantaneous velocities of water and bubble flows on the horizontal plane after a breaking wave front has passed over site2 ((a): $z=-4 \mathrm{~cm}$, wave trough level, (b): $z=-12 \mathrm{~cm}$, bottom level, $t / T=$ 0.340 , red: water velocity, blue: bubble velocity).
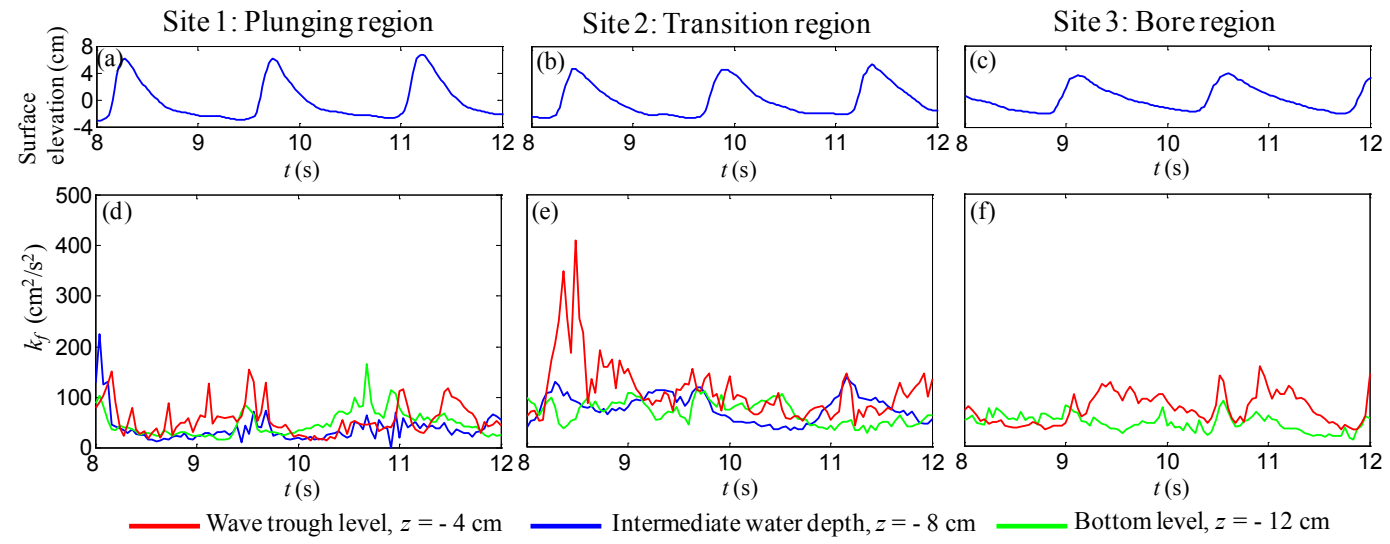

Figure 11. Surface elevation and spatial averaged fluid turbulent energy at site 1 (a) (d), site 2 (b) (e), site 3 (c) (f).

wave generation, $\mathrm{CO}_{2}$ can easily dissolve from the gas phase into water. Gas transfer velocity decreases with time and reaches almost constant value in the bore region (green lines in figure 8). The gas transfer velocity in the transition region is faster than that in the bore region. In the transition region, three-dimensional vortex structures organized by horizontal rollers and obliquely descending vortices are typically produced (Watanabe et al., 2005). While the entrained bubbles trapped within the vortices are restrained to buoyant over long time, $\mathrm{CO}_{2}$ is supplied into water from the bubbles. Therefore, these vortices and bubbles significantly contribute to increased gas transfer velocity in the transition region. The gas transfer velocity in freshwater is higher than that in seawater.

Figure 9 shows the surface elevation and the instantaneous velocity distribution of water and bubble flows on a vertical plane after a breaking wave front has passed over site 2 (transition region). Since, at the breaking wave front, the water jet (red vectors) of a breaking wave plunges and splashes on a forward water surface, the velocities were unable to be estimated due to a high concentration of bubbles. Behind the breaking wave front, typical upward water flow is distributed from the bottom to the water surface, and the upward bubble flow (blue vectors) is also observed to obliquely distribute in this region. This bubble motion may indicate a two-dimensional projection of obliquely descending vortices as described above.

Figure 10 shows the instantaneous velocity vectors of water and bubble flows on the horizontal plane at $z=-4 \mathrm{~cm}$ (wave trough level), $z=-12 \mathrm{~cm}$ (bottom level) after a breaking wave front has passed over site 2 (transition region). It is found that the entrained bubbles are involved in rotating flow generated by an obliquely descending vortex and are transported toward the shore. Since large bubbles 


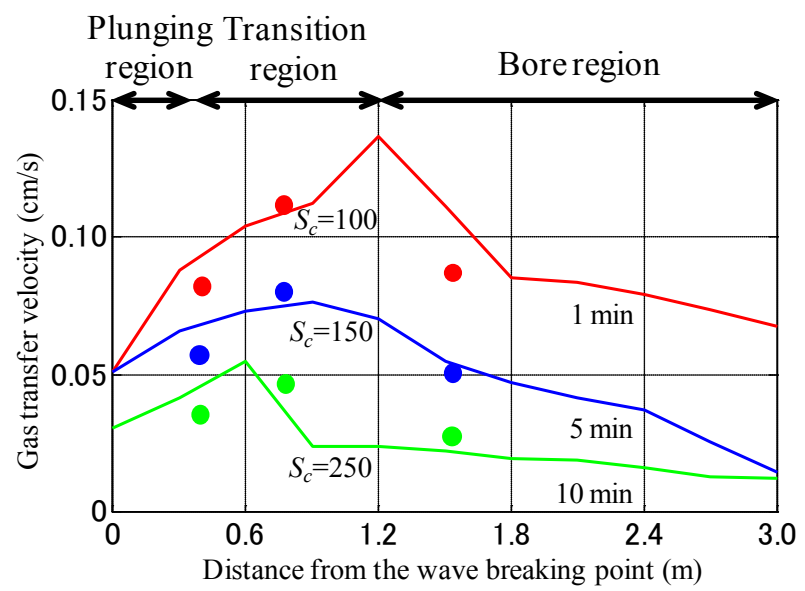

Figure 12. Temporal variations in the cross-shore distribution of gas transfer velocity obtained from Eq. 4 (solid lines) and Eq. 5 (filled circles) (red: $1 \mathrm{~min}$, blue: $5 \mathrm{~min}$, green: $10 \mathrm{~min}$ after the first wave generation).

tend to quickly become buoyant for a short duration, only smaller bubbles can be transported to depths and drift over long time (see Figure10 (b)). Therefore, these smaller bubbles might contribute to enhance the $\mathrm{D}-\mathrm{CO}_{2}$ concentration in a deeper region.

Figure 11 shows the time series of the surface elevation and spatial averaged turbulent energy of water flow in all measurement sites. In the transition region (site 2), the turbulent energy sometimes increases after the passage of breaking waves at $z=-4 \mathrm{~cm}$ (wave trough level) due to the strong turbulent flow generated by obliquely descending vortices. On the other hand, this intermittent feature of the turbulent energy is found to be reduced in the plunging region (site 1) and bore region (site 3 ).

In this study, we tried to estimate gas transfer velocity from the turbulent energy in breaking waves $U_{k}(\mathrm{~cm} / \mathrm{s}) . U_{k}$ was defined by:

$$
U_{k}=\frac{\sqrt{k_{a}}}{S_{c}}
$$

where $k_{a}\left(\mathrm{~cm}^{2} / \mathrm{s}^{2}\right)$ is the temporal and spatial averaged turbulent energy at the wave trough level and, $S_{c}$ is the Schmidt number.

Figure 12 shows the temporal variations in the cross-shore distribution of the gas transfer velocity obtained from Eq. 4 (red, blue and green lines) and Eq. 5 (filled circles). The color lines in Figure 12 correspond to the solid color lines shown in Figure 8(a). It was found that the gas transfer velocity in a surf zone can be roughly estimated from the turbulent energy in breaking waves and the Schmidt number. To develop an appropriate model of the gas transfer velocity in a surf zone, the Schmidt number should be determined by some parameters including water temperature, gas dissolution from entrained bubbles, etc.

\section{CONCLUSIONS}

We measured the temporal variations in the cross-shore distribution of $\mathrm{D}-\mathrm{CO}_{2}$ concentration in a laboratory wave flume using a glass electrode $\mathrm{CO}_{2}$ meter, and also measured the air-water two-phase turbulent flow field in a surf zone using a PIV system with an optical filtering technique. D-CO2 concentration increases with time and the bore region reaches a saturated state earlier than the transition region. The gas transfer velocity in the transition region is much higher than that in the bore region since the numerous entrained bubbles trapped within three-dimensional vortices significantly contribute to the gas dissolution into water. $\mathrm{D}-\mathrm{CO}_{2}$ concentration and the gas transfer velocity in freshwater tend to be higher than those in seawater. Since large entrained bubbles become buoyant after a short duration, only smaller bubbles can be transported to depths. These smaller bubbles might have a significant role to increase $\mathrm{D}-\mathrm{CO}_{2}$ concentration in the deeper area. The gas transfer velocity in a surf zone was estimated from the spatial and temporal averaged turbulent energy in breaking waves and the Schmidt number. It was found that the gas transfer velocity could be roughly estimated from the turbulent energy and the Schmidt number. To develop an appropriate model for gas transfer velocity in 
a near-shore breaking wave field, the Schmidt number should be determined by some parameters including water temperature, gas dissolution from entrained bubbles, etc.

\section{ACKNOWLEDGMENTS}

Financial support for this study was provided by JSPS Research Fellowships for Young Scientist 1902425

\section{REFERENCES}

Liss, P. S. and Merilvat, L. 1986. The role of air-sea exchange in geochemical cycling, Air-sea gas exchange: introduction and synthesis, Dordrecht: Reidel, 133-127.

Melville, W. K. 1996. The role of surface-wave breaking in air-sea interaction, Annual Review of Fluid Mechanics, 28, 279-321.

Nadaoka, K., Hino, M. and Koyano, Y. 1989, Structure of the turbulent flow field under breaking waves in the surf zone, Journal of Fluid Mechanics, 204, 359-387.

Wanninkhof, R. 1992. Relationship between gas exchange and wind speed over the ocean, Journal of Geophysical Research, 97, 7373-7381.

Watanabe, Y., Otsuka, J. and Saruwatari, A. 2008, Laser induced fluorescence measurements of carbon dioxide dissolution in wave-breaking turbulence, Proceedings 31th International Conference on Coastal Engineering, ASCE, 51-59.

Watanabe, Y., H. Saeki. and R. J. Hosking, 2005, Three-dimensional vortex structures under breaking waves, Journal of Fluid Mechanics, 545, 291-328. 